\title{
Letter to Editor: Lamprecht et al. Inflammatory responses to three modes of intense exercise in Standardbred mares - a pilot study
}

\author{
David C. Poole* \\ Department of Anatomy and Physiology, College of Veterinary Medicine, Kansas State \\ University, Manhattan, KS 66506, USA \\ *Corresponding author: poole@vet.ksu.edu
}

Lamprecht et $a l^{1}{ }^{1}$ recently compared the effects of three different exercise tests on heart rate (HR) responses, plasma nitrite concentration and physiological markers of joint inflammation. Even allowing that this was described as a pilot study and therefore may demand less rigorous scientific scrutiny and controls, I wish to take umbrage with several key issues regarding study design, data collection and interpretation. If unchallenged, this publication may do the scientific community a disservice.

Paramount among these issues are: (1) absence of a control group. Particularly, with such a small number of animals $(n=4)$ and the lack of any power analysis, it is crucial to know the underlying variability of the key measurements within the population. Proper experimental controls are essential for making sound scientific comparisons, and these would be essential in any study that utilizes repeated blood sampling and repeated invasive arthocentesis, since these procedures in separatum could have induced an inflammatory response rather than the exercise per se. Specifically, one key outcome and the basis for a (or the) primary conclusion was the plasma nitrite concentration, and yet perusal of Table 2 suggests that the variability of 'Pre-' [nitrite] was the main cause of the very high 'end-' [nitrite]. This concern erodes confidence in the conclusions regarding differences in plasma [nitrite] among exercise conditions. (2) No primary data for the actual HR responses are presented. What were the 'maximal HRs' for these horses? Even allowing for HR not always bearing an immutable relation to oxygen uptake, the ability of HR responses to discriminate among the exercise tests examined is questionable. For instance, Table 3 indicates no significant difference among time spent $>90 \% \mathrm{HR}_{\max }$. If correct, how is the statement in the Abstract 'Mares spent more time at greater than $90 \%$ of $H R_{\max }$ during $R S E T$ [repeated sprint exercise test] than for either GXT [graded exercise test] or IET [interval exercise test]' justified? Oxygen uptake as a measurement of metabolic rate would have improved this investigation substantially. In addition, if there was really 'an effect of exercise test [on] plasma [nitrite]', as stated in the Abstract, it was probably not mediated by (or relative to) any difference in the HR response to the different paradigms. (3) Examining the delta [nitrite] pre-versus end-in Fig. 2, the IET response appears very similar to that for RSET with the principal difference, as mentioned above, being the pre- values. Moreover, although the paper repeatedly uses nitric oxide and nitrite levels synonymously (cf. hypothesis statement in Introduction with last sentence of Abstract), they are not the same thing. There is a complex relationship between $[\mathrm{NO}]$ and [nitrite], with the removal of nitrite itself having a multifaceted kinetics within and among fluid compartments.

In conclusion, the data, as presented, do not provide indisputable support for either a substantially different qualitative or quantitative response in the primary outcome measurements among test types, nor would this be expected if the HR responses presented in Table 3 are a valid measure of exercise stress. One might also question whether this repeated $2 \mathrm{~min}$ bout running paradigm conducted largely at absolute submaximal running speeds (RSET) could, or would, translate to a greater exercise challenge or degree of metabolic stress than one specifically designed to evoke a maximal HR response (IET). Irrespective of these points and the questionable experimental design and data interpretation presented in this paper, the final and concluding statement in their Abstract, 
'As bypotbesized, the bigher-intensity exercise test (RSET) resulted in greater ... markers of exercise intensity compared with lower-intensity exercise tests (IET, GXT)' contains a perverse, somewhat taulological, yet irrefutable, logic.

\section{Reference}

1 Lamprecht ED, Bagnell CA and Williams CA (2009). Inflammatory responses to three modes of intense exercise in Standardbred mares - a pilot study. Comparative Exercise Physiology 5: 115-125. 